\title{
Rappers em São Paulo: conexões, disconexões e transgressões ${ }^{1}$
}

\author{
Charles Kirschbaum- Insper Instituto de Ensino e Pesquisa/CEM-CEBRAP ${ }^{2}$
}

\begin{abstract}
Resumo
Um "campo organizacional" pode ser descrito como uma rede social de atores que estão envolvidos no monitoramento recíproco. Nosso objetivo principal é investigar em que medida os atores sociais dedicam atenção semelhante, dependendo das categorias sociais que eles partilham. Foram entrevistados 35 rappers na cidade de São Paulo, Brasil. Ao longo das entrevistas, mostramos aos nossos entrevistados uma lista de 246 grupos de rap de destaque no Brasil. Para cada grupo, perguntamos aos nossos entrevistados se poderiam apresentar-se com esse grupo no mesmo concerto. Como resultado, obtivemos uma lista de possíveis laços entre os rappers. Nossa análise gerou uma "rede consenso", juntamente com a análise das semelhanças entre os entrevistados, de acordo com suas respostas. Coletou-se o estilo de rap que melhor representava cada entrevistado. Além do estilo, foram coletadas variáveis de controle como idade, tempo no campo, educação formal, renda e repertório. A educação formal, renda e repertório foram significantes, independentemente da afiliação do entrevistado. As afiliações a igrejas pentecostais e ao estilo Underground foram positivas e significantes. Foram geradas agregações parciais das respostas com base nessas categorias, a fim de aprofundar-se a análise da forma como grupos de entrevistados diferem em seus padrões associativos.
\end{abstract}

Palavras-chave: Redes Cognitivas, Redes Sociais, Bonding - Linking - Bridging, Identidade Social

\begin{abstract}
An "organizational field" might be depicted as a social network of actors who are engaged in reciprocal monitoring. Our major goal is to probe the extent that social actors devote similar cognitive attention depending on the social categories they share. We interviewed 35 rappers in the city of São Paulo, Brazil. Throughout the interviews, we showed to our respondents a list of 246 prominent rap groups in Brazil. For each group, we asked our interviewees who could play with that group at the same concert. As a result, we obtained a list of possible (cognitive) ties among rappers. Our analysis generated a consensus network, along with the analysis of similarities among interviewees vis-à-vis their answers. We collected the rap style that best represented each interviewee. In addition to the rap category, we collected control variables like age, time in the field, formal education, income, and repertoire. Formal education, income, and repertoire were significant, regardless of

\footnotetext{
${ }^{1}$ Gostaria de agradecer a Ana Carolina Andrade, Ann Mische, Eduardo Marques, Gazi Islam, Neha Gondal, Paul DiMaggio, Paula Montero, Randall Smith e Ronaldo de Almeida pelas sugestões e provocações em versões anteriores desse artigo. A falha em incorporar suas sugestões é de minha inteira responsabilidade. Essa pesquisa não teria sido possível sem o apoio de Ana Carolina Andrada e André Simões, e a ajuda financeira do Insper Instituto de Ensino e Pesquisa, CEM-Cebrap, CNPq, FAPESP e CAPES em diversos pontos críticos do projeto. Finalmente, gostaria de agradecer aos rappers que se disponibilizaram para conversar conosco.

2 charlesk1@insper.edu.br
} 
the respondent's affiliation. Affiliation to Pentecostal churches and to the Underground rap style were positive and significant. Partial aggregated answers based on these categories were generated in order to further explore how respondents' groups differ in their associational patterns.

Key-words: Cognitive network, Social Networks, Bonding - Linking - Bridging, Social Identity

\section{Introdução}

Pesquisas em Ciências Sociais vêm apontando no Rap um canal de expressão importante, principalmente para jovens afro-descendentes de periferia (CALDEIRA, 2006). Tradicionalmente descrito como movimento cultural, o Rap gera novos espaços de criação e expressão de identidades de estratos populacionais segregados. Entretanto, seus integrantes enfrentam de forma ambivalente as alternativas de integração com o restante da sociedade (Estado, mídia, indústria fonográfica, igreja, etc.) no momento de demarcação das fronteiras identitárias. Dessa forma, proponho o entendimento do Rap como "campo" (BOURDIEU, 1985): as tensões internas entre os vários grupos integrantes do Rap revelam a heterogeneidade e a dimensão relacional no processo identificatório.

Esse trabalho explora as dinâmicas internas do campo do Rap, especialmente como os rappers dão sentido às suas redes sociais e, ao mesmo tempo, intermediam grupos separados por clivagens sociais. Nesse estudo, proponho a investigação do processo identificatório no Rap, seguindo uma abordagem relacional, onde os entrevistados foram levados a identificar as díades legítimas. As "estruturas cognitivas" individuais (estabelecidas a partir de entrevistas com rappers) são comparadas à "rede consensual" (KRACKHARDT, 1987). Essa comparação ilumina os seguintes aspectos ligados à disposição individual no estabelecimento de laços, em relação aos outros entrevistados: (1) medida em que os grupos são percebidos de forma coesa, (2) grau em que os grupos são percebidos como isolados ou centrais, (3) disposição do indivíduo em promover a intermediação de grupos desconexos, a despeito das fronteiras identitárias existentes.

A partir da identificação das características mais salientes na explicação da concordância no reconhecimento das associações possíveis, construo redes "agregadas" dos respondentes que atendem a esses critérios. Em especial, exibo nesse artigo as redes agregadas de respondentes afiliados ao rap Underground e rappers adeptos ao Pentecostalismo. A partir desses dois grafos, mostro como as visões de conectibilidade e desconexão são diversas para esses dois grupos, sugerindo que a forma como cada grupo entende a topologia social pode levar a heurísticas associativas distintas. 


\section{Enquadramento Teórico}

\subsection{Redes Sociais: objetivo e subjetivo}

O estudo de redes sociais (ARS) vem ganhando força e tornando-se central não só nas Ciências Sociais, mas também em várias disciplinas "exatas", como a biologia e a física. A aproximação da Sociologia à Física via ARS traz possibilidades de "fertilização cruzada" entre campos, mas também traz o desafio da reflexão epistemológica. Por exemplo, Granovetter (2003) discute até que ponto a abordagem dos físicos sobre redes sociais pode ser simplesmente transportada para a Sociologia, sem uma reflexão da dimensão interpretativa da ação social que sustenta estruturas como "pequeno mundo" ${ }^{3}$.

Quando nos permitimos afastar de uma visão "objetivista" de redes sociais, abrimos ao mesmo tempo oportunidades, mas introduzimos desafios importantes. Por um lado, é possível conceber o "vínculo" como algo percebido e não necessariamente dado. Krackhardt (1987) propôs que colocássemos a existência de um vínculo como contingente à percepção de uma coletividade de atores. A validade desse construto recai sobre o pressuposto de que em organizações sociais com fronteiras mais ou menos bem definidas existe convergência cultural suficiente para que os vínculos consensualmente percebidos possam corroborar a realidade organizacional. Em contrapartida, os atores individuais variam de acordo com a acuracidade $^{4}$ com que reconhecem como existentes os vínculos que a coletividade estabelece. Dessa forma, Krackhardt constrói uma ponte entre os níveis individuais e coletivos de análise. Estudos recentes vêm elaborando a possibilidade de heterogeneidade cultural, implicando o deslocamento da ideia geral de "acuracidade" para "esquemas interpretativos locais" 5 .

\subsection{Agência e Conexões}

O desafio que proponho nesse artigo é entender as implicações de possíveis mudanças na literatura tradicional de Estruturas Sociais Cognitivas. Assume-se que a percepção das relações está calcada na observação de interações passadas e na

\footnotetext{
${ }^{3}$ Essa discussão pode ser encontrada em diversas tentativas de diálogo entre a Sociologia e a Biologia.

${ }^{4}$ Marsden (2005), ao revisar a literatura oriunda do trabalho de Krackhardt, propõe a distinção entre estruturas "consensuais" (obtidas através das respostas dos entrevistados) e estruturas "reais" (obtidas de fontes externas ao instrumento de coleta de dados relacionais com os entrevistados, como, por exemplo, arquivos ou observação direta). Ao introduzir essa distinção, Marsden propõe que a possibilidade dos indivíduos em perceber a estrutura "real" seja definida como "competência". Nesse movimento, Marsden reafirma a preocupação objetivista de estabelecimento da "rede", como entidade real e externa aos indivíduos, enquanto mantém a salvaguarda da ação social calcada na percepção individual das interações. Ou seja: a percepção subjetiva das relações (dimensão cognitiva) é uma função da observação das interações dos indivíduos (dimensão comportamental).

${ }^{5}$ Ver Kumbasar, Romney e Batchelder (1994).
} 
implicação que interações futuras tomarão como dadas e estáveis essas relações, em detrimento de novas relações que poderiam ser propostas ${ }^{6}$. Ou seja: o que poderíamos aprender sobre a orientação agêntica relacional quando nos focamos nos vínculos potenciais e não apenas nas interações passadas? Essa ideia tem inspiração na tradição de interacionismo simbólico, especialmente de Mead, Cooley e Randall Collins, em conjunto com elaborações recentes da relação entre agência e estrutura.

Se a "sociedade está na mente" (Cooley) e o pensamento pode ser representado como "conversa internalizada" (Mead), podemos nos perguntar de que forma as novas conexões observadas são antecipadas na mente dos indivíduos. A representação mental de "alianças" e "conflitos" entre indivíduos corresponde à combinação e recombinação simbólica das ideias correspondentes a esses objetos concretos (COLLINS, 2004). No nível individual, o esforço de estabelecimento do eu em relação a esses outros internalizados é o fio condutor da formação identitária (DUBET, 1994). Ainda que nos mantenhamos no nível individual de análise, devemos buscar os elementos relacionais que suportam a pré-disposição de estabelecimento de conexões. Ou seja, podemos nos perguntar se existe uma "gramática" de formação de laços, que descreva as relações possíveis e aquelas sancionadas. Essa indagação torna-se importante principalmente quando investigamos em que condições os indivíduos estarão pré-dispostos a ultrapassar as fronteiras identitárias estabelecidas (ou imaginadas) e criar a possibilidade de diálogo fora de seu círculo imediato ${ }^{7}$.

Podemos conceber a dimensão agêntica do estabelecimento e manutenção de vínculos como simultaneamente reprodutora do observado, legitimado e institucionalizado, mas, ao mesmo tempo, da experimentação com vínculos que tragam algum grau de descontinuidade em relação aos laços observados no passado $^{8}$. Assim, interessa-nos entender de que forma os indivíduos variam na prédisposição em repetir as associações sancionadas ou em arriscar a propor vínculos que possam transgredir uma reprodução dos laços anteriores.

\subsection{Cultura, Esquemas e Redes}

Cultura pode ser pensada como articulada à atividade cognitiva de categorização e organização de objetos. Por exemplo, D’Andrade (1995) estudou como indivíduos

\footnotetext{
6 Em contrapartida, veja, por exemplo, Obstfeld (2005). Estudos recentes que coletam dados longitudinais tentam dar uma resposta a esse desafio. No entanto, creio ser distinta a inferência da heurística individual a partir da observação do comportamento ao longo do tempo em comparação com a pré-disposição individual manifestada nas entrevistas.

7 Mische, 2006.

8 Essa intuição é análoga à ideia de agência como temporalmente imbricada (EMIRBAYER e MISCHE, 1998). Ver também Mische (2010).
} 
de culturas distintas associavam doenças de formas distintas ${ }^{9}$. O caráter relacional desse material cognitivo traz à "sociologia da cultura" a oportunidade de investigar esses padrões de associação através do ferramental analítico de ARS. Por exemplo, Yeung (2005) propôs a utilização de látices para analisar "redes de afiliação" que combinavam sentimentos e conceitos diversos em comunidades distintas. Smith (2007) propôs a utilização de ARS para o entendimento de como comunidades distintas compartilham eventos em narrativas conflitantes. Proponho a transposição da intuição de que "membros de culturas distintas associam objetos de formas distintas" para o contexto de formação de vínculos entre pares.

\section{Estratégia Analítica e Métodos}

A análise se inicia com a escolha de "objetos" a serem associados pelos respondentes. No contexto em questão, esses objetos são os próprios rappers proeminentes no campo do hip hop brasileiro. A partir da associação desses rappers entre si, é possível obter para cada respondente uma "fatia" relacional. Em seu conjunto, todas as "fatias" possibilitam a extração de uma rede "consenso" de igual tamanho. Ao comparar a "rede consenso" com as "fatias" individuais, é possível obter uma métrica da forma como os respondentes se assemelham em suas respostas.

Utilizo atributos individuais para explorar possíveis explicações para os padrões de semelhança entre os indivíduos ${ }^{10}$. Os atributos mais salientes (estatisticamente significantes) servem como critérios de agregação dos dados relacionais, com o propósito de construir perspectivas associadas a grupos específicos. Em posse desses grafos, analiso como os respondentes agrupados de formas distintas percebem as relações possíveis entre os rappers proeminentes da lista inicial.

\subsection{Amostra}

Entre 2007 e 2010, entrevistei 35 rappers de municípios da Grande São Paulo. Entre eles, 30 homens e 5 mulheres. Dez entrevistados disseram frequentar templos pentecostais. Perguntei também o "estilo" de rap tocado pelo

\footnotetext{
9 D'Andrade (1995) evoca o conceito de "esquema" para explicar de que forma os objetos são associados pelos indivíduos. Esquemas são concebidos como locais, mas potencialmente compartilhados por grupos.

${ }_{10}$ Por um lado, uma abordagem estritamente relacional poder ser avessa à subordinação dos padrões de relação aos atributos individuais (EMIRBAYER, 1997). Por outro lado, a ideia de "grupo" ganha maior força na medida em que é possível evocar categorias que representem o grupo (BOURDIEU, 1986). Na medida em que os indivíduos podem participar de vários grupos distintos (SIMMEL, 1955), teremos pontos de vista distintos, dependendo do critério de agregação adotado. Alternativamente, eu poderia ter adotado uma abordagem puramente relacional e agrupado os respondentes apenas em função da semelhança de suas respostas. O problema dessa abordagem é que a semelhança entre dois respondentes é altamente influenciada pelo "repertório" ou o número de rappers identificados e arcos associados a esses indivíduos. Ao utilizar o MRQAP, foi possível controlar a semelhança espúria entre respondentes (ver seção 3.5 abaixo).
} 
entrevistado ${ }^{11}$ : 15 se classificaram como "Gangsta", 4 como "pop/comercial", 4 como "hip hop/ rap de raiz", 6 como Gospel ${ }^{12}$ e 6 underground.

Os rappers de minha amostra apresentam uma renda média de mil e duzentos reais (com desvio-padrão relativamente alto, de mil reais). Em média, trinta e dois por cento da renda dos entrevistados provinha de atividades ligadas ao rap (com um desvio-padrão de 43\%). Finalmente, em média, os entrevistados estavam há 10,6 anos no campo do rap (e respectivamente 2,9 anos de desvio-padrão).

\subsection{Coleta de dados relacionais}

Para cada entrevistado, mostrei uma lista de 246 rappers proeminentes da cena musical brasileira ${ }^{13}$. Para cada rapper exibido dessa lista, perguntei "se você fosse um produtor de shows, com quem ' $X$ ' poderia tocar na mesma noite?"14. Foi dada completa liberdade ao entrevistado para realizar "livre associação". Ou seja, não impus nenhum critério ex-ante para a associação de indivíduos aos músicos citados. Com isso, meu objetivo era de colocar o próprio critério de associação como uma pergunta de pesquisa ${ }^{15}$.

\footnotetext{
11 Nos pré-testes às entrevistas busquei a "autoclassificação" dos rappers de forma mais direta, simplesmente perguntando aos rappers qual tipo de rap faziam. Corroborando DiMaggio (1987), obtive muitas vezes categorias nativas idiossincráticas que valorizavam o que havia de único no respondente. Ou então havia simplesmente uma resistência em se autoclassificar. Dessa forma, optei por mostrar pares de rappers proeminentes associados a cada uma das categorias de rap que já havia identificado com informantes para os respondentes e pedir que ranqueassem os "blocos" de acordo com a preferência para tocar junto. A categoria subjacente ao primeiro bloco escolhido foi identificada como uma Proxy à autoclassificação, portanto, uma "autoclassificação indireta".

12 É interessante notar que apenas um subconjunto dos rappers afiliados a seitas pentecostais tocam rap gospel.

${ }^{13}$ Considerei como "proeminente" qualquer rapper que tenha gravado um CD. Justifico esse critério da seguinte forma: se um rapper alcançou um nível mínimo de reconhecimento de seus pares e de seu público, ele será estimulado a gravar um CD para que possa divulgar seu trabalho de forma mais sistemática. Esse critério traz alguns possíveis vieses. Em primeiro lugar, com a ascensão de novas mídias e formas de distribuição de música, o "CD" não é necessariamente o único índice de proeminência. Por outro lado, é possível que vários selos tenham gravado rappers pouco populares com o propósito de completar o "portfólio de estilos". Embora a lista fosse fixa, a ordem dela era sempre aleatorizada, com o propósito de neutralizar o viés de memória dos entrevistados. Em alguns casos, o entrevistado estava incluído nessa lista; entretanto, buscamos uma amostra que não fosse enviesada para rappers já estabelecidos.

${ }^{14} \mathrm{Em}$ alguns casos, o respondente afirmava "poderia tocar com todos rappers". Nesses casos, sugeri que não seria possível colocar "em uma mesma noite" todos os rappers. Se mesmo assim o entrevistado pedisse um limite de grupos, eu sugeria cinco grupos. Dessa forma, tentei, ao máximo possível, não introduzir nenhum viés estabelecendo a priori o número de indicações. Na fase de pré-teste do questionário, perguntamos "quem que a audiência de $X$ gostaria de escutar com ele". Essa pergunta alternativa enviesa a resposta de várias formas, pois não permite a heterogeneidade programada (LAHIRE, 2006), ou não leva em consideração audiências muito amplas.

${ }^{15}$ Existem vários pontos relacionados ao método que são relevantes no momento de interpretação dos resultados, e podem orientar a pesquisa futura. Em primeiro lugar, em contraste com a abordagem tradicional de Krackhardt, eu não pedi aos entrevistados que considerassem todas as díades possíveis entre os rappers da lista original. Isso seria um exercício extenuante. Uma das implicações do uso de "lista livre" é que diminui a probabilidade de concordância entre respondentes. Dessa forma, podemos acreditar que o viés introduzido por essa escolha é conservador. Outra crítica possível é relacionada ao conteúdo dos vínculos e consequentemente à estabilidade do construto da "rede consenso". Ao permitir livre associação, os respondentes podem ser levados a interpretar a pergunta de forma muito distinta. Enquanto um respondente pode preferir vínculos que sejam "sucesso comercial", outro respondente pode favorecer vínculos que promovam similaridade de ideias. Mesmo que eu perguntasse "quais são os rappers mais similares a $X^{\prime \prime}$, esse problema continuaria, pelo caráter multidimensional da homofilia. Uma
} 


\subsection{Atributos Individuais Coletados dos Entrevistados}

Além dos dados relacionais, coletei uma gama de atributos dos entrevistados. Entre as variáveis coletadas, serão incluídas nessa análise: Gênero, Afiliação Pentecostal (dicotômica), Tempo no campo de rap (anos), Renda (reais), Percentual da renda do rap, Educação Formal (anos), Estilo (variável categórica) e Principais Influências (variável relacional ${ }^{16}$ ).

\subsection{Resultados da Análise de Consenso}

A partir das respostas individuais de associação entre rappers descrita na seção acima, selecionei apenas os laços entre os nós incluídos na lista original de rappers proeminentes ${ }^{17}$. Dessa forma, foi possível comparar as respostas dos entrevistados, pois todas as matrizes resposta tinham o mesmo tamanho. A partir da análise de "consenso"18 entre as respostas de todos os entrevistados, foi possível obter a "rede consenso" e os testes estatísticos convencionais para esse tipo de análise. 0 primeiro "autovalor" (eigenvalue) foi de 0,717, enquanto o segundo "autovalor" foi de 0,492 . Esses dois valores geram uma razão baixa, apenas de 1,458 , o que sugere que os respondentes não "compartilham de uma mesma cultura". A "competência média" dos respondentes foi de 13,8\% (desvio-padrão de 3,7 \%).

\subsection{Modelo Multivariado MRQAP.}

\footnotetext{
das possibilidades seria fixar um tipo de vínculo, como por exemplo, similaridade discursiva. Em muitos casos, os entrevistados revelavam durante o exercício que era esse o critério que estavam utilizando para compor as díades. Mas, ao fixar esse critério, eu perderia os vínculos baseados em outros critérios, como "batida semelhante". Quando abordamos esse problema do ponto de vista de "patamar" (threshold) necessário para o estabelecimento do vínculo (MARSDEN, 2005), nos preocupamos com falsos positivos e falsos negativos. Falsos negativos (ausência de vínculo) não deveriam ser o foco da preocupação: a "livre associação" gera uma coesão baixa entre os nós e traz um grau maior de conservadorismo para nossas conclusões. Talvez o mais preocupante sejam os falsos positivos. A pergunta atual pode levar a dois respondentes indicar um vínculo entre dois rappers sem que concordem com os critérios subjacentes ao estabelecimento desse vínculo. A abordagem atual, no entanto, me parece melhor que as alternativas. Se, por exemplo, eu perguntasse "quais são os rappers semelhantes a $X^{\prime \prime}$, eu poderia obter associações que não levassem em consideração conflitos não subordinados à obra artística. Uma das possibilidades aventadas e experimentadas foi questionar o entrevistado sobre suas razões para estabelecer o vínculo. Em muitos casos, o respondente não sabia precisar o porquê, apontando apenas para uma afinidade. Assim, é possível que, dado um critério pré-estabelecido pelo entrevistador, o respondente seja capaz de sugerir vínculos que atendam ao requisito, mas o inverso não é necessariamente verdade - a sugestão de um vínculo toma uma dimensão "pré-cognitiva" ou pelo menos "pré-analítica", o que nos aproxima de uma visão de "paradigma de grupos mínimos" (Minimal group paradigm, ver Tajfel, 1970). Gostaria de agradecer ao meu colega Gazi Islam por essa última sugestão.

${ }^{16}$ Coletei durante as entrevistas os rappers que mais haviam influenciado o respondente. A agregação das respostas (respondentes e influências) gerou uma matriz afiliação.

17 Ou seja: nomes citados que não estavam na lista original foram registrados, mas não foram incluídos nessa análise.

18 Essa análise assemelha-se bastante a uma análise fatorial, onde o estabelecimento do laço consensual é dado pelas respostas de todos os respondentes. Ver Romney, Weller e Batchelder, 1986 para a proposição teórica original. Krackhardt (1986) operacionalizou essa proposta combinando-a com a análise de redes sociais. Eu utilizei o software Ucinet para realizar essa análise (ver BORGATTI, EVERETT, FREEMAN, 2002), com os seguintes parâmetros: Type of data: "Networks: Square nonsymmetric matrix for each respondent"; Analytic model: "Interval".
} 
Utilizei o algoritmo MRQAP (multiple regression quadratic assignment procedure) ${ }^{19}$, com o propósito de identificar as variáveis que melhor explicam concordância entre os respondentes. Assim, a variável dependente foi a matriz de concordância entre os respondentes, vis-à-vis a rede consensual. A seguir, descreverei as matrizes que correspondem às variáveis independentes incluídas nessa análise.

- Mesmo estilo: $\mathrm{X}_{\mathrm{i}, \mathrm{j}}=1$ se $i$ e $j$ são associados ao mesmo estilo; caso contrário, $\mathrm{X}_{\mathrm{i}, \mathrm{j}}=0$ (uma matriz por categoria, Underground, Pop, Gangsta, Gospel, Hip hop e uma matriz sem distinção de categorias, chamada de "Rótulo Categórico")

- $\quad$ Diferença de Tempo no campo: $X_{i, j}$ é igual ao módulo da diferença entre o tempo no campo de i e $j$; padronizado

- $\quad$ Produto de Tempo no campo: $\mathrm{X}_{\mathrm{i}, \mathrm{j}}$ é igual ao produto do tempo no campo de $\mathrm{i}$ e j; padronizado

- Diferença de Renda: $X_{i, j}$ é igual ao módulo da diferença entre a renda de i e j; padronizado

- $\quad$ Produto de Renda : $\mathrm{X}_{\mathrm{i}, \mathrm{j}}$ é igual ao produto da renda de $\mathrm{i}$ e $\mathrm{j}$; padronizado

- $\quad$ Percentual de Renda do Rap: $X_{i, j}$ é igual ao módulo da diferença entre o percentual da renda oriunda do rap de i e j; padronizado

- Diferença de Educação formal: $X_{i, j}$ é igual ao módulo da diferença entre a Educação Formal de i e j; padronizado

- $\quad$ Produto de Educação formal: $\mathrm{X}_{\mathrm{i}, \mathrm{j}}$ é igual ao produto da Educação Formal de $\mathrm{i}$ e j; padronizado

- $\quad$ Principais Influências: $X_{i, j}$ é distância euclidiana quando transformamos a matriz afiliação de Influências na matriz quadrada de respondentes (ver BREIGER, 1974).

\footnotetext{
${ }^{19}$ Essa análise se assemelha a uma regressão múltipla, mas a variável dependente é o vínculo entre os atores. Dessa forma, tanto a variável dependente quanto as independentes são matrizes quadradas de mesmo tamanho (ver DEKKER, KRACKHARDT, SNIJDERS, 2007). MRQAP é adequado quando as variáveis independentes não são elas mesmas influenciadas pela variável dependente; ou seja, esse método é inadequado para situações onde há endogenia, e, portanto, as variáveis independentes não podem incluir métricas de centralidade, por exemplo.
} 
Variáveis de Controle.

Além das variáveis independentes identificadas acima, incluí variáveis de controle que podem estar relacionadas com as semelhanças de respostas entre os entrevistados:

- Repertório ${ }^{20}$ (número de grupos que o entrevistado reconheceu da lista original): $X_{i, j}$ é igual ao produto do repertório de i e $j$.

- Mesmo Gênero: $X_{i, j}=1$ se $i$ e $j$ são associados ao mesmo gênero; caso contrário, $\mathrm{X}_{\mathrm{i}, \mathrm{j}}=0$

- $\quad$ Afiliação Pentecostal $=X_{i, j}=1$ se $i$ e $j$ expressam afiliação pentecostal; caso contrário, $\mathrm{X}_{\mathrm{i}, \mathrm{j}}=0$

\subsection{Identificação de Grupos e Triangulação com os Atributos dos Respondentes}

A extração da "rede consenso" pode sugerir fronteiras identitárias que devem ser respeitadas por toda a comunidade de rappers ${ }^{21}$. Utilizei algoritmo proposto por Girvan e Newman 22 para identificar "comunidades" a partir da coesão entre nós na rede consenso. Por outro lado, dependendo da "afiliação" do rapper, ele pode ver as fronteiras de forma mais rígida ou mais flexível ${ }^{23}$. Dessa forma, agreguei as respostas dos respondentes a partir das variáveis mais significativas identificadas nos modelos MRQAP, para gerar redes parciais.

\section{Resultados e Análises}

Modelos MRQAP. Na tabela 1 apresento dois modelos MRQAP. O Modelo 1 utiliza todas as variáveis independentes e de controle descritas acima. O Modelo 2 retém apenas as variáveis estatisticamente significantes ${ }^{24}$.

\footnotetext{
20 Ver Swidler (1986) para a ideia de cultura como uma "caixa de ferramentas" (repertório). Em um estudo mais recente (SWIDLER, 2001), o "volume" do repertório traz implicações para a forma como o indivíduo interpreta as diferentes situações onde se encontra.

${ }^{21}$ Ver Lamont e Molnar (2002).

22 Ver Girvan e Newman (2002).

${ }^{23}$ Ver Zerubavel (1991) para uma discussão sobre culturas mais "rígidas" e "flexíveis".

${ }^{24}$ Obtive o Modelo $2 \mathrm{com}$ um procedimento "bootleg" convencional. A cada rodada eu eliminava a variável com maior $\mathrm{p}$-valor.
} 
Variáveis

$\underline{\text { Modelo } 1} \underline{\text { Modelo } 2}$

\begin{tabular}{lcc}
\hline Mesmo estilo & & \\
$\quad$ Rótulo Categórico & 0,120 & \\
Underground & 0,154 & $0,225 * * *$ \\
$\quad$ Pop & $-0,013$ & \\
Gangsta & $-0,046$ & \\
$\quad$ Gospel & $-0,068$ & \\
$\quad$ Hip hop & $-0,002$ & \\
Tempo no campo & $-0,045$ & \\
Produto de Tempo no campo & $-0,086$ & \\
Diferença de Renda & $-0,118 *$ & $-0,143 * *$ \\
Produto de Renda & $-0,041$ & \\
Diferença de Educação formal & $0,132 * *$ & $0,130 * *$ \\
Produto de Educação formal & 0,049 & \\
Principais Influências & $-0,039$ & \\
\hline Repertório & $0,360 * * *$ & $0,333 * * *$ \\
Mesmo Gênero & $-0,031$ & \\
$\quad$ Feminino & $-0,048$ & $-0,069 *$ \\
$\quad$ Masculino & 0,135 & \\
Afiliação Pentecostal & $0,094 *$ & $0,119 *$ \\
& & \\
& & \\
$R^{2}$ Ajustado & & \\
Probabilidade & $19,9 \%$ & $18,5 \%$ \\
$*$ p $<0.1 ; * *$ p $<0.05 ; * * * p<0.01 ; \mathrm{N}=1190$ & $0,0 \%$ \\
\end{tabular}

Tabela 1: Resultados da Análise MRQAP

É interessante notar que, de todas as categorias de estilo, apenas a autoclassificação ao Underground mostrou-se significante. Esperaríamos que Gospel, ao impor fronteiras identitárias fortes, levaria a um grau alto de semelhança de reconhecimento de vínculos entre seus membros. No entanto, uma vez que inserimos a variável de controle "Afiliação Pentecostal", a variável "Gospel" mostrou-se estatisticamente não significante, sugerindo que a identificação com o pentecostalismo leva a padrões de atenção e padrões de vínculos semelhantes, mas não necessariamente à adesão ao rap Gospel.

A variável "Diferença de Renda" está negativamente associada com a semelhança de padrões de vínculos entre os pares. Essa relação é intuitiva e pode ser apoiada pela sociologia do consumo cultural, onde diferenças de renda estão associadas tanto ao acesso quanto à afirmação de status social ${ }^{25}$. Em contraste, a variável

${ }^{25}$ Ver Bourdieu (2006) para um desenvolvimento teórico robusto sobre o tema. Nesse artigo não me estendo sobre a discussão contemporânea sobre a "distinção", mas ver DiMaggio e Muhktar (2004) para um estudo empírico mostrando o declínio do consumo cultural como implicação de acúmulo de capital cultural. 
"Diferença de Educação formal" está positivamente associada com a semelhança de padrões de vínculos entre os pares. Esse resultado é, prima facie, contraintuitivo, no sentido em que esperaríamos que diferenças de educação formal também influenciassem tanto o acesso à obra artística ${ }^{26}$, quanto a predisposição de distinção. Entretanto, a correlação de Educação Formal e Renda é de 0,23, sugerindo que Renda seja interpretada como variável moderadora entre "Educação Formal" e "concordância".

É interessante notar que a variável gênero, especificamente a matriz "Feminino", obteve um parâmetro negativo, embora com baixa significância27. Quando examinamos a tabela de correlações, a variável "feminino" tem correlação negativa com a variável dependente "concordância", novamente com baixa significância, sugerindo a robustez do resultado obtido na análise multivariada.

Finalmente, como esperado, a variável de controle "Repertório" apresentou um parâmetro positivo e altamente significante, sugerindo que quanto maior o número de grupos associados a alters, maior a chance de indicação dos mesmos alters.

Partições Girvan-Newman. A figura 1 traz o grafo da rede consenso com os nós já identificados de acordo com as partições extraídas pelo algoritmo Girvan-Newman. É interessante notar que o grupo de nós cinza e nós com formato de quadrado se destacam como uma "ilha" em potencial. Esses nós são geralmente identificados como rappers Gospel. De forma menos acentuada, o grupo de nós brancos com formato triangular no canto superior do grafo é geralmente identificado com rappers Underground. Ambos os grupos mostram-se salientes também nos modelos MRQAP. No centro, os nós com formato redondo e preto, temos o grupo "Mainstream" que agrega rappers Gangsta, Pop e de raiz ${ }^{28}$.

\footnotetext{
26 Ver Bourdieu e Darbel (2007).

${ }^{27}$ Por limitações de espaço, a dimensão de "gênero" não será abordada nessa versão.

${ }^{28}$ Uma das constatações importantes é a ausência de hiato entre pop, gangsta e rap de raiz. De forma contrária a vários estudos na sociologia da arte que insistem na polarização entre produtores "autênticos" e "comerciais", aqui existe clara possibilidade de articulação, a despeito das "farpas" generosamente compartilhadas nas letras atuais (ROSE, 2008; PECQUEUX, 2009). Mas veja a obra de Thaide para uma visão bem menos polarizada.
} 


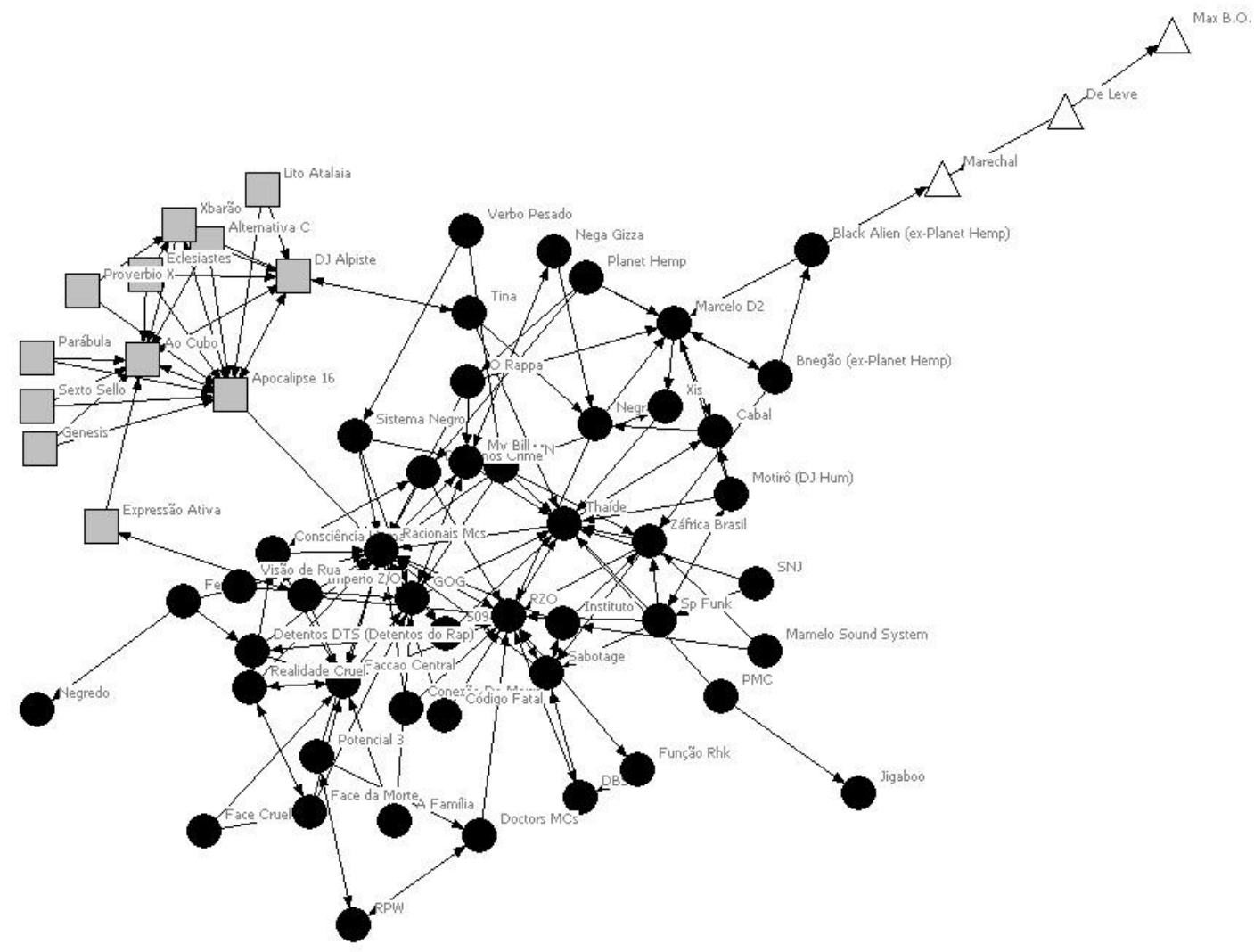

Figura 1: Rede Consenso e Agrupamentos Girvan-Newman

A Figura 2 traz as respostas agregadas de todos os respondentes afiliados ao Pentecostalismo. Eu mantive os nós com as cores respectivas às partições obtidas sobre a rede consenso, para facilitar a comparação. Além disso, estabeleci como parâmetro de "força de laço" o número de respondentes que concordavam com a existência díade. É interessante que encontramos as duas partições "Gospel" e "Mainstream" agora colados, sem o hiato verificado na rede consenso. Além disso, identificamos laços fortes no interior do grupo Gospel, assim como no grupo Mainstream. 


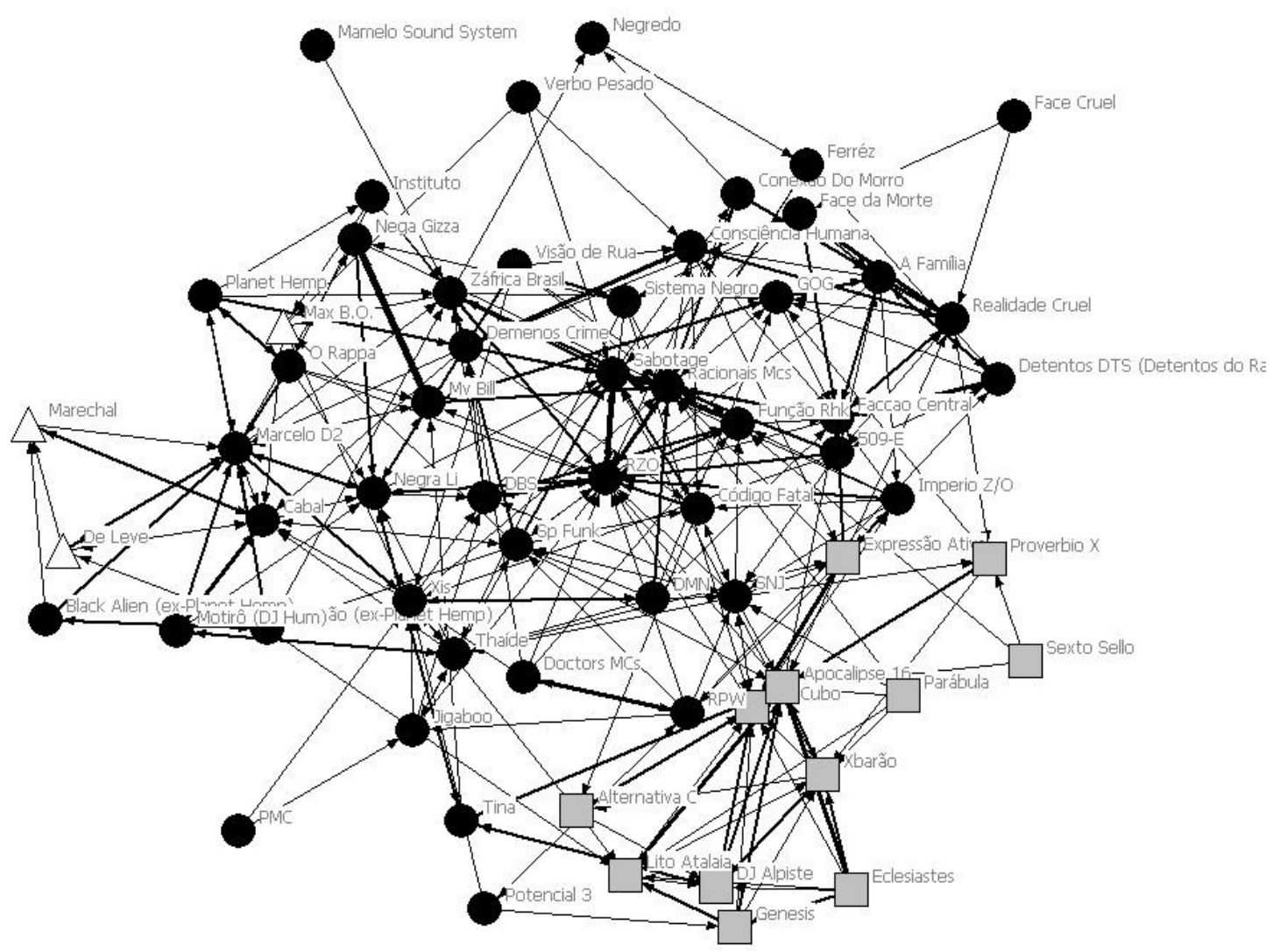

Figura 2: Respostas Agregadas de afiliados ao Pentecostalismo

A Figura 3 traz o grafo de respostas agregadas dos respondentes identificados como Underground. Em contraste com a Figura 1, os nós identificados com o Underground estão completamente integrados no bloco Mainstream. Em contraste, os nós Gospel são identificados como fracamente ligados ao Mainstream. 


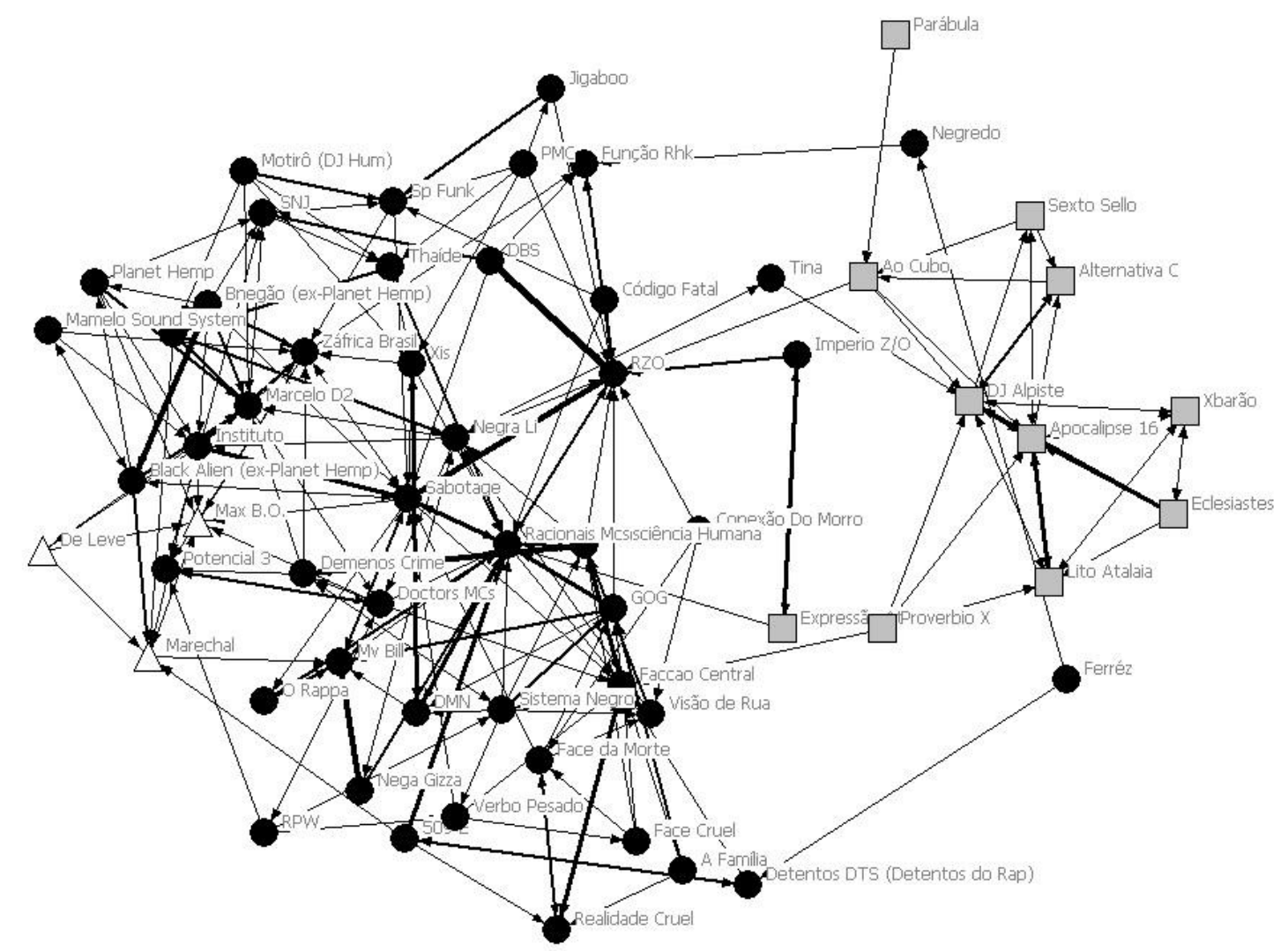

Figura 3: Respostas Agregadas de afiliados ao Underground

\section{Discussão e Conclusões}

A preocupação central desse paper é de propor uma metodologia que pudesse, ao mesmo tempo, identificar a "topologia" relacional de um campo, mas preservar as lógicas locais na explicação de como essa topologia emerge.

Ao examinar tanto a "rede consenso" quanto a análise MRQAP, verificamos que há dois grupos mais salientes - Gospel e Underground. Curiosamente, a afiliação ao pentecostalismo mostrou-se mais importante para explicar a concordância entre os padrões de associação. Essa é uma situação onde os padrões relacionais percebidos não estão subordinados à afiliação categórica ${ }^{29}$. Quando examinamos a Figura 2, onde havia uma integração relativamente maior entre rappers Gospel e rappers mainstream, podemos inferir que a possibilidade de desacoplagem entre pentecostalismo e rap gospel permite a "transgressão" da fronteira identitária que havíamos identificado na rede consenso.

Poderíamos então nos perguntar: se os rappers gospel não impõem, eles próprios, a desconexão observada na rede consenso, onde ela se origina? Quando examinamos a Figura 3, vemos o grupo de rappers gospel fracamente conectado ao

\footnotetext{
${ }^{29}$ DiMaggio, 1993.
} 
restante dos indivíduos relacionados. Uma das implicações dessas observações é que a forma como um grupo se vê no espaço social pode não corresponder à forma como é visto pelos outros ${ }^{30}$. Essa assimetria nos padrões associativos pode ser vivenciada como um estigma. Por outro lado, ao tornarmos a força das fronteiras identitárias dependente da percepção local, é possível evitar o tratamento reificado das fronteiras identitárias: uma vez que as fronteiras dependem da forma como os atores implicados às percebem, possíveis transgressões tornam-se mais aceitáveis ${ }^{31}$.

Versões posteriores dessa pesquisa poderão incorporar dados relacionais externos às pesquisas junto aos rappers, com o objetivo de entender não só "acuracidade", mas também "competência" dos entrevistados ${ }^{32}$. Além disso, será oportuna a incorporação de laços negativos (de rejeição), o que poderá ajudar na interpretação das desconexões e hiatos e a incorporar de forma mais direta a dimensão conflituosa.

Creio ser interessante voltar a enfatizar, nessa conclusão, a importância do movimento rap para jovens oriundos de regiões periféricas e desfavoráveis na formação de suas identidades e no acesso aos espaços públicos. Não são raros os relatos que apontam a necessidade de união desse movimento. Mas, de forma concomitante, observa-se que a pluralidade de discursos e posicionamentos torna a coesão do movimento um desafio constante para seus integrantes (Buzo, 2010). Os resultados desse artigo podem iluminar aspectos subjacentes à ação de maior integração no rap.

\section{Referências}

Alves, C. (2004). Pergunte a Quem Conhece: Thaíde. São Paulo: Labortexto.

Borgatti, S. P., Everett, M. G., \& Freeman, L. C. (2002). Ucinet for Windows: Software for Social Network Analysis. Harvard, MA: Analytic Technologies.

Bourdieu, P. (1985). The Social Space and the Genesis of the Groups. Theory and Society, $14,723-744$.

Bourdieu, P. (1986). The Forms of Capital. Handbook of Theory and Research for the Sociology of Education (p. 241-58). Westport: Greenwood Press.

Bourdieu, P. (2006). A Distinção: a crítica social do julgamento. São Paulo: Edusp.

${ }^{30}$ Compare com Elias, 2000, onde se assume uma simetria em como os "outsiders" se vêem e são vistos.

31 Pachucki, Pendergrass e Lamont, 2007.

32 Essa abordagem pode ser importante como oportunidade de checagem da rede "consenso" obtida unicamente a partir das respostas dos entrevistados. 
Bourdieu, P., Darbel, A., Schnapper, D., \& de Freitas Teixeira, G. J. (2007). O amor pela arte: os museus de arte na Europa e seu público. EdUSP.

Breiger, R. L. (1974). The Duality of Persons and Groups. Social Forces, 53, 181190.

Buzo, A. (2010). Hip-hop: dentro do movimento. Rio de Janeiro: Aeroplano Editora.

Caldeira, T. P. R. (2006). I Came to Sabotage Your Reasoning! Law and Disorder in the Postcolony (p. 102-149). Chicago: Chicago University Press.

Collins, R. (2004). Interaction Ritual Chains. Princeton, NJ: Princeton University Press.

D'Andrade, R. G. (1995). The Development of Cognitive Anthropology. Cambridge, UK: Cambridge University Press.

Dekker, D., Krackhardt, D., \& Snijders, T. A. B. (2007). Sensitivity of MRQAP Tests to Collinearity and Autocorrelation Conditions. Psychometrika.

DiMaggio, P. J. (1987). Classification in Art. American Sociological Review, 52, 440455.

DiMaggio, P. (1993). Nadel's Paradox revisited: Relational and cultural aspects of organizational structures. In N. Nohria \& R. Eccles (Orgs.), Networks and organizations: structure, form, and action (Networks and organizations : structure, form, and action.). Boston: Harvard Business School Press.

DiMaggio, P., \& Mukhtar, T. (2004). Arts Participation as Cultural Capital in the United States, 1982-2002: signs of decline? Poetics, 32, 169-94.

Dubet, F. (1994). Sociologia da experiência. Inst. Piaget.

Elias, N. (2000). Os estabelecidos e os outsiders. Rio de Janeiro: Jorge Zahar, 1950 .

Emirbayer, M. (1997). Manifesto for a Relational Sociology. American Journal of Sociology, 103, 281-317.

Emirbayer, M., \& Mische, A. (1998). What is Agency. Am. J. Sociology, 103, 9621023.

Girvan, M., \& Newman, M. E. J. (2002). Community structure in social and biological networks. Proceedings of the National Academy of Sciences of the United States of America, 99(12), 7821.

Granovetter, M. S. (2003). Ignorance, Knowledge, and Outcomes in a Small World. Science, 301, 773-774. 
Krackhardt, D. (1987). Cognitive Social Structures. Social Networks, 9, 109-134.

Kumbasar, E., Rommey, A. K., \& Batchelder, W. H. (1994). Systematic biases in social perception. American Journal of Sociology, 477-505.

Lahire, B. (2006). A cultura dos indivíduos. Porto Alegre: Artmed.

Lamont, M., \& Molnar, V. (2002). The study of boundaries in the social sciences. Annual Review of Sociology, 28, 167-195.

Marsden, P. V. (2005). Recent developments in network measurement. Models and methods in social network analysis, 8, 30.

Mische, A. (2006). Partisan Publics: Contention and Mediation across Brazilian Youth Activist Networks. Princeton: Princeton University Press.

Mische, A. (2010). Relational Sociology, Culture, and Agency. Sage Handbook of Social Network Analysis (p. 67-81). Thousand Oaks, CA: Sage.

Obstfeld, D. (2005). Social Networks, the Tertius Iungens Orientation, and Involvement in Innovation. Administrative Science Quarterly, 50, 100-130.

Pachucki, M. A., \& Breiger, R. L. (2010). Cultural Holes: Beyond Relationality in Social Networks and Culture doi:10.1146/annurev.soc.012809.102615. Annual Review of Sociology, 36, 205-224.

Pachucki, M., Pendergrass, S., \& Lamont, M. (2007). Boundary processes: Recent theoretical developments and new contributions. Poetics, 35, 331-351.

Pecqueux, A. (2009). Le Rap. Paris: Le cavalier bleu.

Romney, A. K., Weller, S. C., \& Batchelder, W. H. (1986). Culture as Consensus: A Theory of Culture and Informant Accuracy. American Anthropologist, 88, 313-338.

Rose, T. (2008). The Hip-Hop Wars: What We Talk about When We Talk about HipHop--and Why It Matters. Basic Civitas Books.

Simmel, G. (1955). Conflict and The Web of Group-Affiliations. Glencoe, IL: The Free Press.

Smith, T. (2007). Narrative boundaries and the dynamics of ethnic conflict and conciliation. Poetics, 35, 22-46.

Swidler, A. (1986). Culture in Action: symbols and strategies. American Sociological Review, 51, 273-286.

Swidler, A. (2001). Talk of Love: How Culture Matters. Chicago: Chicago University Press. 
Tajfel, H. (1970). Experiments in intergroup discrimination. Scientific American, 223(5), 96-102.

Yeung, K. (2005). What does love mean? Exploring network culture in two network settings. Social Forces, 84, 391-420.

Zerubavel, E. (1991). The Fine Line: making distinctions in everyday life. New York: Free Press. 